\section{Surface Layers on Tungsten produced by Active Nitrogen.}

WE have found that a fine tungsten filament at a very dull-red temperature placed in a stream of nitrogen undergoes a considerable (10-25 per cent.) lowcring of its resistance (for constant current flowing through it) when active nitrogen produced up-stream by a condensed discharge passes over it. At the same time the colour changes to a much duller red, that is, the radiation is decreased. These changes persist until the filament is momentarily flashed at a white heat, whereupon the original condition is regained. Apparently a surface layer of some sort forms on the filament and produces a lowering of its temperature. The resistance of the filament at room temperature is the same either with or without the layer.

The same cooling effect can be produced by bombarding still nitrogen with electrons from another source filament accelerated by suitable electric fields. In a tube with large nickel electrodes which had been thoroughly degassed by the repeated use of an induction furnace, this effect was first detectable when the exciting electrons had an energy of 11 volts. The rate of formation of the layer increased rapidly with the voltage. In a second tube, in which the anode was a hot tungsten spiral, the effect was not detectable below 22 volts. The effect was independent of the potential of the testing filament, showing it to be produced by a neutral substance. It seems possible that in the first case the active substance was produced at the low voltage by bombardment of the metal surfacos. These effects were obtained both with commercial nitrogen which had been passed over hot copper and with nitrogen prepared by Waran's method which was so pure that it would not give the afterglow.

Peculiar current-voltage characteristics were obtained with the tube having the hot anode. With increasing voltage the current increased, as in other gases, up to 22 volts, at which voltage it began to fall off. At 25 volts an are struck, the current took a sudden riso immediately, followed by a decrease in two or three seconds to a value considerably below that which it had had before the arc struck. Further increases in voltage produced slight increases in current. Upon decreasing the voltage, the current dropped off until the are broke at about 20 volts. Here there was a sudden decrease in current followed by a slower rise to a much higher current, the same as that at the corresponding voltage before the arc had struck. The two currents were the same for lower voltages. That those effects are characteristic of nitrogen and not ascribable to charges on the walls of the tube seems probable, for no such effects were observed with argon in the tube. A decrease of the thermionic current in nitrogen at much lower pressures and higher voltages was observed by Langmuir (Phys. Rev., 2, p. 450; 1913). Wo also observed a considerable temporary decrease in the thermionic emission from a tungsten filament as ordinary active nitrogen passed over it.

A tube was constructed having a pile of tungsten foil discs, spaced apart, and mounted so that they could be flashed with the induction furnace. Electrodes and filaments were provided, so that the nitrogen could be activated either by the disruptive discharge or by electron bombardment of known voltage. Gas pressures were measured with a hot wire guage of small volume. The total volume of the tube and gauge was small enough in relation to the area of tungsten that a gas layer one atom deep on the latter, if evaporated, would cause a pressure of the order of $0.1 \mathrm{~mm}$. The tube was baked out under exhaust, as usual, at $450^{\circ} \mathrm{C}$. and the discs well degassed by flashing. With a few tenths of a millimetre of nitrogen in the tube, and kecping the dises at a dull-red heat while the spark discharge was passed, a partial clean-up of the gas was obtained. After exhausting the remaining gas and closing off the pump, the dises were flashed, with the discovery of a considerable quantity of gas. Gas so recovered was not cleaned up by a hot tungsten filament, and on examination with a hand spectroscope gave all evidences of being nitrogen. The experiment was repeated a large number of times, varying tho time of the spark discharge. In all cases except where the time of discharge had been very short, the amount of gas recovered on flashing was a constant quantity, approximately that to be expected from a layer one atom deep. Exactly the same results were obtained by activating the nitrogen with an are at 25 volts. At 15 volts there was no indication of clean up, or of a gas layer on the tungsten which could be removed by flashing.

Our conclusions are that a clean tungsten surfaco at a dull-red heat, if placed in an atmosphere of nitrogen, activated either by a condensed discharge or by an electron bombardment at more than 22 volts, bocomes covered with a nitrogen layer of the order of one atom deep. The effect of this layer, at this comparatively low temperature, is to cool the surface. It seems probable that it does this by allowing the surface to conduct more heat to the gas, i.e. by increasing the accommodation coefficient. At relatively high tem. peratures, the same layer is probably so unstable that only a small fraction of the surface can be covered at any one time, but it acts to increase the work function. The flashing of a filament covered with such a layer in the neighbourhood of a clean filament causes the production of a layer upon the latter. Apparently the layer evaporated by flashing comes off in an active form. These experiments suggest that active nitrogen can bo produced by bombardment of nitrogen gas with 22 volt electrons, but further work is necessary to establish this conclusion. The experiments are being extended and completed, and will be described in detail later.

CARL Kenty.

Louis A. Turner.

Palmer Physical Laboratory,

Princeton University,

Princeton, New Jersey, U.S.A.

\section{The Temperature Variation of the Elasticity of Rochelle Salt.}

VAlasek (Phys. Rev,, 478; 1922) has studied the temperature variation of the piezo-electric modulus of Rochelle salt. He found abrupt change in the values of this modulus at temperatures of $-15^{\circ} \mathrm{C}$. and $23^{\circ} \mathrm{C}$., using crystal slabs with their length at $45^{\circ}$ with the $b$ and $c$ crystallographic axes. In a later paper he describes experiments undertaken to investigate the temperature variation of other physical properties of the crystal, but these experiments do not indicate discontinuities such as occurred in the case of the piezo-modulus.

The object of this note is to point out that there is evidence for the existence of similar discontinuitics in the values of the elastic constants of this crystal at these two temperatures.

Slabs of Rochelle salt have been cut and mounted so as to form piezo-resonators, similar to the quartz piezo-resonators used in modern wireless practice. As shown by Cady and by Dye, these piezo-resonators possess a well-defined resonant frequency, which depends on the dimensions, the density, and the Young's modulus of the crystal. Hence, by in- 